\title{
On the Nature and Composition of Abstract (Theoretical) Concepts: The X-Ception Theory and Methods for Its Assessment
}

\author{
Luigi Pastore, Sara Dellantonio, Claudio Mulatti and Remo Job
}

\begin{abstract}
The 'standard picture of meaning' suggests that natural languages are composed of two different kinds of words: concrete words whose meaning rely on observable properties of external objects and abstract words which are essentially linguistic constructs. In this study, we challenge this picture and support a new view of the nature and composition of abstract concepts suggesting that they also rely to a greater or lesser degree on body-related information. Specifically, we support a version of this new view which we call "x-ception theory" maintaining that abstract concepts are based on internal information of a proprioceptive, interoceptive and affective kind. Secondly, we address a methodological issue concerning the so-called concreteness and imageability measures, two tools that are widely used in (mainly psycholinguistic) empirical research to assess the degree of concreteness of specific words. On the basis of this analysis we argue that-even though the classical concreteness and imageability measures were developed in relation to the standard picture of meaning - they can also be used in the new framework of x-ception theory. In particular, we suggest that the discrepancy between these two measures provides a clue as to whether a word relies on internal information. By contrast, we argue that a new measure for concreteness recently proposed in order to address some problems with the old measure is completely inappropriate for this aim.
\end{abstract}

L. Pastore

Università degli Studi di Bari, Bari, Italy

e-mail: luigi.pastore@uniba.it

S. Dellantonio $(\varangle) \cdot$ R. Job

Università degli Studi di Trento, Trento, Italy

e-mail: sara.dellantonio@unitn.it

R. Job

e-mail: remo.job@unitn.it

C. Mulatti

Università degli Studi di Padova, Padova, Italy

e-mail: claudio.mulatti@unipd.it

(C) Springer International Publishing Switzerland 2015

L. Magnani et al. (eds.), Philosophy and Cognitive Science II,

Studies in Applied Philosophy, Epistemology and Rational Ethics 20,

DOI 10.1007/978-3-319-18479-1_3

全 337249_1_En_3_Chapter-online $\checkmark$ TYPESET $\square$ DISK $\square$ LE $\square$ CP Disp.:14/5/2015 Pages: 58 Layout: T1-Standard 


\title{
1 Introduction
}

The 'standard picture of meaning' suggests that natural languages are composed of two different kinds of words: concrete words "whose meaning are fixed by their relations with observable properties of the environment" and abstract words whose meanings are "fixed by a network of inferential or other relations to the meanings of other words, including those belonging to the observation vocabulary." (Cruse 2000, p. 52). According to this picture, these two word classes are structurally different from each other since abstract words are linguistic (i.e. purely definitional) constructs, while concrete words are based on perceptual information driven by the external world. Words that do not rely on perceptual information are organized in a structure of growing abstraction, in which terms based more directly on observational vocabulary are considered more concrete than those which rely on other linguistic constructs.

However, recent studies carried out mainly in the field of the so-called embodied cognition challenge this view and suggest that abstract words are not just linguistic constructs, and that at least some of them do rely on sensory information that is not driven by external perception, but rather concerns internal states of the body. As e.g. Barsalou states: "Recent embodiment theorists propose that knowledge acquired from introspection is central to the representation of abstract concepts." (Barsalou 2008, p. 620; for an overview see also e.g., Barsalou 1999; Barsalou et al. 2003; Barsalou and Wiemer-Hastings 2005). The view that abstract words rely (at least to some extent) on introspective information challenges this standard picture of meaning and reshuffles the cards of the classical architecture of concrete and abstract words.

In fact, some authors suggest that there is no strict opposition between concrete and abstract words, and that apparent differences result from the fact that words refer to concepts that are composed in different proportions of external sensory information, internal experience and linguistic information. For example Vigliocco and collaborators maintain:

\begin{abstract}
The apparent dichotomy between concrete and abstract word meanings arises because of a statistical preponderance for sensory-motor information to underlie concrete word meanings and a preponderance for affective and linguistic information to underlie abstract word meanings. While sensory-motor information is statistically more preponderant for concrete word meanings, affective and linguistic information is statistically more important for abstract word meanings both for their acquisition and their subsequent representation in the adult system. (Vigliocco et al. 2009, p. 223; see also Kousta et al. 2011).
\end{abstract}

Not only has the standard picture of meaning been challenged by this view, but also the two measures psycholinguistics operationalized in order to distinguish between concrete and abstract words - the so-called 'concreteness' and 'imageability' constructs - seem to have become obsolete with respect to this debate, since they rely on the classical notion of concreteness as something based only on external perception.

In this paper we address both the theoretical and the methodological aspects of this issue. First of all, we discuss this new idea of 'abstractness' in comparison to the old one and we argue that word meanings reflect different degrees of involvement of 
different kinds of external and internal sensory information as well as of linguistic information, positioning words in different parts of a multidimensional space that allows clusters of words to be closer together on any of these dimensions. Specifically, we support a version of this new idea of 'abstractness' which we call 'x-ception theory' (Dellantonio et al. 2014) according to which abstract concepts rely to a greater or lesser extent on internal information of a proprioceptive, interoceptive and affective kind. This theory allows us - among other things - to give a more precise idea of what kind of abstract concepts might be based more heavily on internal information or might, on the contrary, rely on this information only to a minimal extent.

Secondly, we address a methodological issue concerning the so-called 'concreteness' and 'imageability' constructs, i.e. the main scales that have been defined to measure concreteness versus abstractness. Specifically, we will shed light on the definition of concreteness versus abstractness they are implicitly built on and illustrate what they really measure. On the basis of this analysis we argue that-even though the classical concreteness and imageability measures have been developed in relation to the standard picture of meaning - they can also be used in the new framework of $\mathrm{x}$-ception theory. Specifically, we suggest that the discrepancy between the two offers a clue to assess whether a word relies on internal information. By contrast, we argue that a new measure for concreteness recently proposed in order to address some problems with the old measure, is completely inappropriate for this aim.

83

\section{The Standard Picture of Abstractness}

The classical view of abstraction proposed by both the philosophical and the psychological research defines abstract concepts (or words) ${ }^{1}$ in opposition to concrete ones: a concept (or word) is considered as concrete if it denotes observable things in the external world, while it qualifies as abstract when it does not refer to something perceivable. This differentiation between concrete and abstract words/concepts is at the basis of the 'standard picture of meaning' according to which natural languages are composed of two structurally different classes of words, i.e. concrete words based on perceptual information "whose meaning are fixed by their relations with observable properties of the environment" and abstract words whose meanings are "fixed by a network of inferential or other relations to the meanings of other words, including those belonging to the observation vocabulary" which are therefore purely linguistic constructs (Cruse 2000, p. 52). Words that do not rely on perceptual information are organized in a structure of growing abstraction, in which those based more directly

${ }^{1}$ In our view, 'concepts' and 'words' are equivalent notions since concepts are considered to be the internal representations that support the semantic competence a person has with respect to the corresponding words. To know a word (i.e. to apply it correctly), one must have a corresponding concept that allows her/him to group together the class of objects denoted by the word. In this sense, concepts and words can be considered as equivalent at least in the sense that they must rely on corresponding inclusion/exclusion criteria (on this see also Dellantonio and Pastore 2006). 
on observational vocabulary are considered more concrete than those which rely on other abstract linguistic constructs positioned higher in the structure.

As far as philosophical investigation is concerned, the standard picture of meaning originates in the field of philosophy of science and specifically from research on the relationship between the observable and unobservable entities in a theory and the nature of the terms describing those entities: the so-called observational terms denoting observable objects and so-called theoretical terms denoting unobservable entities (Carnap 1956; Achinstein 1965; Papineau 1996). There is no clear-cut distinction between theoretical and observational terms, but they are organized in a continuum from the perceptual (i.e. observational) periphery to the highest theoretical level. The more a term is distanced from the observational level the more reference fixing becomes problematic and dependent on the theory, i.e. on the place the term occupies in the corpus of the theory. Thus, theoretical terms depend heavily if not entirely on the definitional and mathematical apparatus of a theory and are related to the observational periphery only though the mediation of other terms. While the observational vocabulary can be considered quite stable intersubjectively, the theoretical vocabulary varies and changes in time together with (i.e. depending on) the theory that defines it.

These considerations regarding the observational and the theoretical vocabulary of a scientific theory apply in the same way to natural languages and to the differentiation between concrete and abstract words. In fact, the issue of theoretical terms addressed in the field of the philosophy of science is actually one and the same as the issue of abstract terms discussed in the field of psychology and, more recently, of philosophy of psychology. Concrete words are conceived as those that refer to perceptible, material entities that can be directly observed. As e.g. Jesse Prinz maintains, 'democracy' is an abstract concept because its referent cannot be directly experienced in perception; because it cannot be seen, heard, smelled, or tasted (Prinz 2002, p. 167). On the contrary, abstract words are analogous to theoretical terms in the sense that they do not have a referent that can be directly perceived, but they depend on a specific definition given by (some kind of) 'theory', which does not need to be a scientific theory, but can also consist in a commonsensical view. Think e.g. of words like 'democracy', 'truth' or 'belief' that are often presented as prototypical examples of abstract terms (see also e.g. Barsalou 1999; Connell and Lynott 2012): people have commonsensical views on the (definitions of) these things which determines the knowledge they have of the corresponding terms. Thus, what we identify as 'truth' or 'democracy' or 'beliefs' and the properties we associate with them depend (implicitly or explicitly) on the definition we acquired. As pointed out also by Alan Paivio - one of the most important authors in psychology who tried to account for the difference between abstract and concrete terms from the point of view of the psychological mechanisms that are supposed to underlie their processing-the philosophical discussion on theoretical terms is closely related to the psychological discussion on abstract words: "My perspective on the issue is pragmatic and psychological. The basic assumption is that the observational-theoretical distinction becomes psychologically real when interpreted in terms of the correlated difference between concrete and abstract terms." (Paivio 1986, p. 11). 
When Paivio says that the distinction between abstract and concrete terms is "psychologically real" he is not just speaking metaphorically to imply that this is psychologically relevant. In fact, he is rather referring to a specific hypothesis he 45 puts forward on the way abstract terms are mentally represented which is called ${ }_{16}$ Dual Coding Theory. At its core, Paivio's Dual Coding Theory suggests that the 47 cognitive system is composed of two different symbolic subsystems connected to ${ }_{8}$ each other: a verbal one, specialized for the processing of language, and a nonverbal one, also called the imagery system, whose function is mainly to perceptually so analyze the external world and generate mental images of it. The word 'imagery' 51 describes both this system and the capacity this system enables to dynamically form and recall mental images. Mental images are not meant as visual pictures only, but basically describe traces stored in memory of all kind of sensations - acoustic, olfactory, haptic and gustatory. In this sense, an 'acoustic image' would be the nonverbal representation of a sound, an olfactory image would be the nonverbal representation of a smell, etc. As Paivio specifies: "Our minds 'contain' memory isomorphs of how entities and events look, sound, feel." (Paivio 2007, p. 25).

According to the Dual Coding Theory, the main difference between concrete and abstract words is that, while concrete words are represented in both the verbal and the nonverbal systems, abstract words are represented in the verbal system only: they depend largely on linguistic information and have only weak referential relationships.

Both classes of words (abstract and concrete) have interconnections with the representations of other words in the verbal system. The specific nature and structure of the verbal-associative networks for concrete and abstract words presumably differ in systematic ways that reflect differences in the contexts in which they have been acquired and used, but in general it can be said that concrete and abstract words are semantically differentiated by the degree of availability of referential interconnections. Concrete words have both referential and verbalassociative meaning, whereas abstract words depend relatively more on verbal-associative interconnections for their meaning. (Paivio 1986, p. 123).

For this reason "comprehension is more dependent on imagery in the case of concrete than abstract sentences." (Paivio 1986, p. 219).

Paivio's hypothesis is part of a more general line of research on the so-called mental imagery which investigates the processes and the mechanisms through which we can mentally recall any kind of sensation and that support capacities that are usually described as 'visualizing', 'seeing in the mind's eye', 'hearing in the head' or 'imagining the feel of something'. Paivio's proposal was-as in general most theories supporting the existence of mental imagery developed in the same years (for an overview see e.g. Nigel 2013) - a reaction against the classical computational view according to which linguistic representations need to be conceived uniquely as abstract, amodal and language-like symbolic structures unrelated with the physical and functional features of the referents and not bound to the perceptual system. Computational views explain conceptual thought and linguistic capacities on the basis of internal relations between symbols, without adequately accounting for the problem of how these are connected to the external world. Imagery is introduced to explain how such amodal symbols are anchored to nonlinguistic perception so that people can understand in a referentially salient sense the meaning of words. In this sense, the main point of 
Paivio's Dual Code might be seen in the problem of understanding how reference works: how people identify what things in the word correspond to a certain word. The implicit idea of a Dual Code is that—even though language understanding is partially due to verbal-associative interconnections and among verbal representations- these verbal-associative interconnections are not enough to account for the human capacity of using words to denote things. Thus, the problem Paivio faces on the basis of his theory is to account for both aspects of language understanding, the capacity to define words through other words and the capacity to connect words with things in the external word.

In this respect, Paivio's theory complies with the standard picture of meaning in suggesting that language is characterized by a dichotomy between terms that do have an observable reference in the external world which can be perceived through the senses and terms that are based on definitions. Thus, both the classical philosophical and psychological views on language and specifically on semantics rely on the idea that the ingredients needed to fix the meaning of terms and to understand them are sensory information and linguistic knowledge only. No other ingredient seemed to be needed. Abstractness and concreteness were defined on the basis of these two kinds of information only and their degree was supposed to depend on their reciprocal weights. This is the 'transdisciplinary mainstream' in opposition to which a new perspective has recently begun to be developed. This new perspective-which we are going to present in the next section-challenges this two-dimensional picture of language and frames a three-dimensional model, which does not only include external sensory information, and verbal-associative information, but also internal bodily experience.

\section{Abstractness in a New Perspective: The X-ception Theory}

The idea that the referential component of meaning can be explained solely in terms of the relationship between words and something observable in the external world is a classical principle not only in psychological but also in philosophical research. As for the latter, there is an influential research tradition arguing that-in order to ensure the intersubjectivity of meaning-reference must consist of something that everybody can observe. If the reference were 'private' and perceivable by a person only, we would never be sure that different people use the same words to indicate the same things (Wittgenstein 1953, 1967, §256ff; Kripke 1982; for an overview on the so-called 'private language argument' see e.g. Cook 1965; Schroeder 1998; Knorrp 2003; Candlish and Wrisley 2012). For this reason, according to this tradition the reference of words cannot be identified using internal sensory experience since this would be accessible only by the first person and stability of meaning couldn't be granted: i.e. it could neither be granted that words have univocal meanings independently of the specific person that uses them, nor that everybody uses them to denote one and the same thing. 
However, the first attempts to assess Paivio's theory indicated that taking the position that reference can be understood uniquely as a relationship between a word and some observable object or property in the external world might not hold. The element in Paivio's theory that immediately presented difficulties was the emotions (specifically, emotion words). In fact, since emotions do not have any observable reference in the external world, they should not be represented in the imagery system, and the meaning of emotion words should be understood uniquely on the basis of verbal-associative interconnections among words. However, in Paivio's study of 1968 he had already pointed out that the situation seems to be more complex than this.

According to Paivio's theory, words that easily evoke sensory information have a direct representation in the imagery system, while words that evoke sensory information only with difficulty are represented solely in the verbal system. Since Paivio hypothesized that only concrete words have a direct representation in the imagery system, he predicted that only this word class should easily arouse sensory information. To confirm this hypothesis, Paivio developed an imageability scale that measures the image-evoking capacity of words meant as their capacity to arouse sensory (i.e. perceptual) experience stored in memory (Paivio 1965; Paivio et al. 1968) and then confronted the ratings obtained using this scale with the concreteness ratings previously collected for the same words by Spreen and Schulz (1966). If Paivio's model made the right prediction, imageability should always reflect concreteness and the two ratings should always be strongly correlated. Paivio et al. (1968) found indeed a high correlation $(0.83)$ which was widely confirmed by later studies. In a recent analysis on the ratings of 4260 words included in the MRC database (Coltheart 1981; Wilson 1988) we confirmed a positive correlation between imageability and concreteness of 0.835 (Dellantonio et al. 2014; in this paper we also report other literature confirming this result). However, in spite of this strong general correlation, the prediction is not entirely confirmed since there are a number of words mainly referring to emotional states for which imageability and concreteness are uncorrelated. For those problematic items, imageability ratings are significantly higher than concreteness ratings. As Paivio observed, these items exhibit interesting similarities:

\begin{abstract}
Most of these are words with strong emotional and evaluative connotations. The largest group consists of terms referring to affective reactions or affective attitudes: AFFECTION, AGONY, AMAZEMENT, AMOUR, ANGER, ANXIETY, DEVOTION, FUN, GAIETY, GRATITUDE, GRIEF, HAPPINESS, HATRED, HOPE, HOSTILITY, HUMOR, INSOLENCE, JEALOUSY, JOVIALITY, JOY, KINDNESS, LOVE, LOYALTY, MISERY, MOOD, PANIC, PASSION, PLEASURE, PRIDE, SADNESS, SHAME. Others in this category are labels for attitudes and emotional situations, or are generally evaluative in meaning: BLESSING, BRAVERY, CHAOS, CHARM, CHRISTMAS, COURTSHIP, DEATH, GLORY, OBEDIENCE, OBSESSION, SAFETY, TRAGEDY and VANITY. (Paivio et al. 1968, p. 7). ${ }^{2}$
\end{abstract}

Analogous inconsistencies were noted in later studies (see e.g. Altarriba et al. 1999; Altarriba and Bauer 2004, p. 397). We were also able to confirm them in a previous study (Dellantonio et al. 2014) conducted on the concreteness and imageability

${ }^{2}$ The few anomalies in the opposite direction are quite easily explained: they involve like 'antitoxin', 'armadillo', 'encephalon', 'dell' which denote concrete objects that are unusual and therefore most of the raters are not familiar with their appearance.

\begin{tabular}{l|l|l|l|}
\hline 337249_1_En_3_Chapter-online $\square$ TYPESET $\square$ DISK $\square$ LE $\square$ CP Disp.:14/5/2015 Pages: 58 Layout: T1-Standard \\
\hline
\end{tabular} 
ratings collected in the MRC database which is one of the most important available sources for imageability and concreteness ratings (Coltheart 1981; Wilson 1988). In this work we selected 36 emotion and moods words taken from a number of studies on basic emotions and moods (Tomkins 1962, 1963; Ekman et al. 1969; Plutchik 1980; Ekman 1994, 1999; Reizenzein 2009; Kassam et al. 2013; Prinz 2004; Damasio 1999) and we compared them with ten randomly chosen control groups. The results showed that imageability ratings were significantly higher than concreteness ratings for emotion/mood words compared to the words in control groups.

The problem that Paivio already identified with respect to these recalcitrant words is that-even though they surely do not denote anything observable and therefore cannot be qualified as concrete - they still rely on some kind of sensory experience: "These words appear to have the common property of having been associated with sensory experience (usually affective in nature) but not specific things or classes of things". (Paivio et al. 1968, p. 7). This point raised by Paivio is as crucial as it is controversial since it suggests that word meanings might not be based on external perception and linguistic information only, but they might also be grounded in another kind of sensory experience, i.e. in affective experience.

If this is correct, then the reason why emotion words have a high imageability (i.e. according to Paivio's definition of imageability: easily arouse sensory experience stored in memory) is that they are represented in the non-verbal, imagery system like concrete words and that they have therefore a referential component, consisting in the affective experience that characterizes their occurrence. In this case it is plausible to assume that the inconsistency between concreteness and imageability ratings in the case of emotion words is not a problem of the theory, but provides a clue to an unexpected situation. Specifically, it may indicate that the understanding of nonconcrete words (i.e. words with low concreteness ratings) like emotion words also relies on sensory experience stored in memory, which is however not of an external, but of an internal kind. In this case, imageability should be interpreted as a measure of the ease/difficulty with which a word evokes both external and internal sensory experience.

As for concrete words, imageability correlates with concreteness because concreteness measures the link between a word and some external sensory information while imageability assesses the easiness/difficulty with which a word evokes this external sensory experience stored in memory. In the case of non-concrete words, imageability does not necessarily correlate with concreteness, because it is possible that - as in the case of emotions - even though a word is not linked to external sensory information, it relies on internal sensory experience. If so, then a discrepancy between imageability and concreteness ratings like that shown by emotion words can be considered an important clue that a word easily evokes internal sensory experience (for a more detailed discussion of this hypothesis see Dellantonio et al. 2014).

This conclusion is consistent with many recent theories, mainly those related to the tradition of embodied cognition which maintain that "knowledge acquired from introspection is central to the representation of abstract concepts." (Barsalou 2008, p. 620; see also e.g., Barsalou 1999; Barsalou et al. 2003; Barsalou and Wiemer- 
Hastings 2005; Lakoff 1987; Johnson 1987; Lakoff and Johnson 1980; Gibbs and Jr 1994; Lakoff and Turner 1989). As Barsalou specifies elsewhere, introspective states "include events perceived inside the mind and body that typically lack counterparts in the external world, such as emotions, affects, appetitive states, cognitive operations, and beliefs." (Barsalou et al. 2003, p. 44 nota). As also e.g. Prinz points out, these states "stem $[\cdots]$ from within the body (as with proprioception, interoception, hunger, and thirst)." (Prinz 2002, p. 116).

One of the most recent attempts made by the psychological (i.e. psycholinguistical) research to theoretically systematize this conception of abstract terms and their mental representation is that proposed by authors like Kousta and Vigliocco (see e.g. Vigliocco et al. 2009; Kousta et al. 2011). According to them, both concrete and abstract words are made of two different types of information: 'experiential information' and 'linguistic information'. The 'linguistic information' is of verbal-associative kind and it corresponds to the one the standard picture relies on. As for 'experiential information', they think that this is of a twofold kind, or more specifically that it is drawn from two different sources depending on whether we are considering concrete or abstract concepts. The experiential information concrete concepts consist in is described as 'sensory-motor information': this notion is meant to indicate perceptual information driven by sensory-motor interactions with the outside world. The experiential information abstract concepts rely on is identified as 'affective information', i.e. information driven by the experience everyone has of their emotions and emotional states. (Vigliocco et al. 2009). As e.g. Vigliocco and collaborators maintain:

The apparent dichotomy between concrete and abstract word meanings arises because of a statistical preponderance for sensory-motor information to underlie concrete word meanings and a preponderance for affective and linguistic information to underlie abstract word meanings. While sensory-motor information is statistically more preponderant for concrete word meanings, affective and linguistic information is statistically more important for abstract word meanings, both for their acquisition and their subsequent representation in the adult system. (Vigliocco et al. 2009, p. 223-italics added)

And further, in the version of this view proposed by Kousta and collaborators:

[...] we propose that both concrete and abstract concepts bind different types of information: experiential information (sensory, motor, and affective) and also linguistic information. However, concrete and abstract semantic representations differ in terms of whether sensory, motor, or affective information have the greatest weight, with sensory-motor information being more preponderant for concrete concepts and affective information playing a greater role for abstract concepts. Thus, a central and novel element of this proposal is the idea that experiential information contributes to the representation of both concrete and abstract words. However, whereas sensory-motor information is statistically more important for the representation of concrete words, emotional content, a largely neglected type of experiential information in the literature on semantic representation/processing, contributes to word representation and processing, particularly for abstract concepts. (Kousta et al. 2011, p. 14 - italics added) 
These quotes show clearly that Vigliocco, Kousta and collaborators use the word 'affective' as a synonym for 'emotional', ${ }^{3}$ thus considering emotional content as the only kind of internal experience abstract concepts rely on. However, affective experience meant in the sense of emotional experiences is only one of the various kinds of internal experience subjects perceive 'introspectively' (on introspection as a kind of perception see Goldman 1993). As implied by Barsalou's and Prinz's quotes reported above, there are a variety of other types of internal information we can introspectively access.

Other types of internal, introspective states very similar to emotions which also rely on sensory experience are those conveyed by proprioception (which provides information on body position, body movements and the muscular system, see e.g., Berthoz 2000) and interoception ${ }^{4}$ (which provides information on the internal condition of the body: monitoring states like heartbeat, respiration, pain, hunger, thirst, the need for digestion, elimination, etc., see Craig 2003, 2009, 2010 for a more specific discussion of proprioception and interoception as well as of their relationship to emotional experience see Dellantonio, Pastore, forthcoming, Chap. $1 \S 5, \S 6) .{ }^{5}$ Since there is no reason to think that affective experience is special with respect to proprioception and interoception and that affective experience is therefore the only relevant,

\footnotetext{
${ }^{3}$ In the philosophical and in the classical psychological tradition, terms like "affect", "affective" or "affection" assume a different meaning. In fact, the word "affection" is derived from Latin terms like affectus and afficere used to translate the Greek term pathos" which indicates the experience of any kind of event or modification caused by the interaction with an entity other than myself. The verb "to affect" in English preserves in part this original meaning. The word is used in this way e.g. by Aristotle in his doctrine of categories (Ackrill 1963). However, in his On the Soul Aristotle also used the word "affection" in another more restricted and specific sense to indicate only the passive modifications of the psyche that occur without the active and voluntary participation of the subject. In this sense "affections" are identified with and described as passions. In the modern era the word "affection" was used in this restricted sense by Descartes (Brown 2006) and Spinoza (Lebuffe 2010). This interpretation of "affection" in the sense of passion is also that inherited by psychological research. Here "affection" indicates not only the passive modifications of the psyche, but also the effects of this alteration (Dixon 2003). Thus, the word implicitly recalls the idea of an effect caused by some modifications. In a non-metaphysical context these modifications must concern primarily the body, thus we should use "affection" to indicate any state which is the effect of some bodily modification. In this sense, interoception and proprioception are univocally particular kinds of affective states while emotions belong to the class of affections and are affective states only insofar as they are caused by bodily modifications. However, this sense of the word has been lost in the contemporary psychological usage of the term in which affective and emotional have become synonyms.

${ }^{4}$ To be more precise, we should distinguish between interoception and nociception, which is the perception of any kind of pain; however, as e.g. Craig $(2003,2009)$ and Damasio $(1999,2010)$ maintain, nociception can be considered a form of interoception.

${ }^{5}$ Interoceptive and proprioceptive information might even be essential for the perception of emotional feelings since we experience our emotions also by means or in virtue of specific corresponding bodily changes: for example we experience fear also by virtue/means of bodily changes such as increased heart and respiration rates, muscular tension etc.; we experience shame also by virtue/means of increased blood flow in the face resulting in facial blushing, gastric and visceral contractions, etc.). On the relationship between emotional information and interoceptive/proprioceptive information see Dellantonio, Pastore, forthcoming, Chap.4).
}

气 337249_1_En_3_Chapter-online $\square$ TYPESET $\square$ DISK $\square$ LE $\square$ CP Disp.:14/5/2015 Pages: 58 Layout: T1-Standard 
internal source of sensory information for the understanding of word meaning, it is plausible to hypothesize that the view proposed by Kousta, Vigliocco and collaborators might be extended at least to interoceptive and proprioceptive experience. This would imply that word meanings might rely on all kinds of internal information people consciously perceive on the internal states of their body including (at least) affection, interoception and proprioception. We called this x-ception theory (Dellantonio et al. 2014).

The idea that the representations of words denoting proprioceptive or interoceptive states relies on the corresponding internal information was the object of a previous study we carried out, in which we analyzed the imageability and concreteness ratings of a set of words included in the MRC database which denote proprioceptive and interoceptive states (Dellantonio et al. 2014). Our statistical analysis confirmed that this word class exhibits properties analogous to emotion words: i.e. it proved that the difference between imageability and concreteness ratings for interoceptive and proprioceptive words is significantly higher than that for the control groups (randomly chosen from the database). Also in this case, the inconsistency between imageability and concreteness ratings might indicate that — even though these words are not linked to external sensory information - they still arouse internal sensory experience.

As a further proof of the hypothesis that the difference between imageability and concreteness can be interpreted as a measure of whether a word representation relies on internal information, another data set analyzed in the previously mentioned study (Dellantonio et al. 2014) is worth reporting. Here we selected a number of theoretical terms relying on the definition of theoretical given by the classical philosophical debate (see §2); specifically, we chose abstract terms belonging to the technical jargon of a discipline whose meaning is highly dependent on the linguistic definition given them by the framework of that theory like e.g. 'axiom' (mathematics); 'causality' (physics); 'conjugation' (linguistics); 'legislation' (politics and law); 'deduction' (logic); or 'theory' (science in general). Our hypothesis suggests that for this particular class of abstract words, no sensible discrepancy between imageability and concreteness should be observed, since this word class should not rely (at least not to a large extent) on internal sensory information. In fact, as in the case of concrete words, their imageability ratings should depend on their concreteness ratings only and the two measures should correlate. In Dellantonio et al. (2014), we found that the differences between imageability and concreteness for the theoretical/technical words are either smaller than, or comparable to, those of the control groups. In addition, we carried out another analysis specifically for this paper confirming that they are also correlated $(\mathrm{r}=0.743, p<0.001)$.

Since the method we developed to assess the internal grounding of words based on the discrepancy between imageability and concreteness ratings gave encouraging results, we used it to provide some new data for this study. Specifically, we tested Barsalou's hypothesis (Barsalou 1999; Barsalou et al. 2003) that our capacity to understand words denoting what can be termed doxastic mental states such as e.g. 'to know', 'to believe', 'to be certain', 'truth', 'false' etc. and words denoting states like 'hope', 'desire', 'wish', 'remember', etc. which we might more properly call attitudes is due, at least in part, to the availability of internal, introspectively 
accessible information for these states. To do so, we analyzed a new group of words included in the MRC database denoting specifically doxastic and epistemic states (e.g. 'true', 'truth', 'right', 'wrong', 'certain' 'uncertain', 'falsehood', 'unknown', etc.). Even though the number of words we were able to include in the analysis is relatively low (16), the analysis showed that the mean difference between imageability and concreteness of doxastic states words (mean difference $=60$ ) is statistically identical to the mean difference between imageability and concreteness of $\mathrm{x}$-ceptive words (mean difference $=72$ ), $\mathrm{t}<1$. More specifically, doxastic words behave like proprioceptive and interoceptive words. ${ }^{6}$

This result is particularly interesting since it shows that there are also other classes of non-concrete words beyond proprioceptive, interoceptive and emotional terms that rely on internal information and this offers some support for the thesis that many if not all abstract (i.e. non-concrete) concepts include some internal information. As further proof of this and to see what kind of concepts are more largely based on internal information we used this method of identifying the discrepancy between imageability and concreteness in the opposite direction. Instead of starting from specific terminological classes selected a priori on the basis of some criteria, we extracted all the words that have the highest discrepancy between imageability and concreteness from the MRC database: for each word in the MRC database provided with imageability and concreteness ratings we computed the difference between imageabiilty and concreteness, we then computed the mean and standard deviation of this set and selected those words for which the difference between imageability and concreteness was 2 standard deviations above the mean of the database. The words selected on the basis of this criterion are interestingly varied, confirming that internal information is crucial not only for the understanding of proprioceptive, interoceptive and emotional terms, but that many word types largely rely on introspective information.

In fact, among the term matching this criterion there are not only the words classes we expected to find on the basis of our results, i.e. words denoting:

- emotions/moods like 'joy', 'jealousy', 'happiness', 'love', 'unhappiness', 'fun', 'optimism', 'terror' etc.;

- interoceptive and propriceptive states such as 'relaxation', 'warmth', 'excitement', 'thrill' etc.;

- conditions that can be considered midway between interoceptive and emotive states such as 'pleasure', 'anxiety', 'tranquil', 'excitement', 'unpleasantness', 'uneasiness' etc.;

- or states closely related to emotions such as 'grief', 'hostility', 'bravery', 'romance', 'intimate', 'danger', 'humor', 'seduction', 'beauty'.

This group of words also includes other kinds of terms that are usually more univocally considered as abstract like words denoting:

${ }^{6}$ In this regard, it should be specified that the mean difference between imageability and concreteness of proprioceptive and interoceptive terms is significantly smaller than the mean difference between the imageability and concreteness of emotion words (mean difference $=119), \mathrm{t}(47)=2.8, p<0.01$. We do not have a conclusive explanation for this result, however see in Dellantonio et al. (2014) for some possible hypotheses.

a 337249_1_En_3_Chapter-online $\square$ TYPESET $\square$ DISK $\square$ LE $\square$ CP Disp.:14/5/2015 Pages: 58 Layout: T1-Standard 
- supernatural/religious phenomena such as 'devil', 'mystery', 'magic', 'ghost', 'eternal', 'devotion', 'goddess', 'demon', 'sin', 'hell', 'angel', 'paradise';

- significant events or times in life such as 'maternity', 'marry', 'graduation', 'marriage', 'holiday', 'adolescence', 'maturity', 'childhood', 'summer';

- interpersonal behaviors related to values such as 'kindness', 'insolence', 'hostility', 'obedience', 'adultery', 'gratitude', 'greed', 'luxury', 'vanity', 'independence', 'pride', 'failure';

- emotionally connoted social relationships such as 'marriage', 'freedom', 'friendship', 'poverty';

- mental states such as 'obsession', 'delirium', 'hope', 'reflection'.

If our interpretation of the imageability measure and of its relationship with the concreteness measure is correct, then these words are strongly related to internal sensory information of some kind. We neither have a specific explanation for why these words seem to be more linked than other words to internal information, nor do we completely trust the accuracy of the measure we use (about this see the next section). However, this result univocally indicates that many words commonly considered as abstract seem-according to the imageability ratings people assign to them-not to be merely linguistic constructs, but to rely on some kind of internal information. What kind of internal information they rely on must be the object of further investigation.

In spite of this open question, the analysis we carried out as well as the data we reported allow us now to draw some conclusions regarding two aspects we considered previously. On the one hand, these findings challenge the classical view of abstract words according to which they must be conceived as theoretical terms, i.e. as purely linguistic constructs. They suggest instead a differentiation of degree between abstract words which are closer to theoretical terms as they are classically defined (those abstract terms whose representations consist almost uniquely of verbal associative information) and abstract words of a different kind, whose representations rely for a large part also on internal sensory experience.

On the other hand, on the basis of our observations there is no reason to maintain that affective experience is the only kind of internal information that plays a role with respect to our understanding of abstract terms, as implicitly suggested by Vigliocco, Kousta and collaborators. On the contrary, our findings lead us to think that all kinds of internal experience introspectively available to the subject might be relevant for understanding (some) non-concrete words. At the very least, proprioceptive and introspective information are surely part of the internal information people rely on to understand some word classes. For this reason we call our view x-ception theory.

By suggesting that a more accurate classification of the internal information people have at their disposal is essential to shed light on the debate on the semantic differences among words and words representations, we propose a view according to which word meanings reflect different degrees of involvement of different kinds of external and internal sensory information as well as linguistic information, positioning words in different parts of a multidimensional space that allows clusters of words to be closer together on any of these three dimensions. In consideration of 
this position, the very notion of reference needs to be revisited, taking into account the possibility that at least some words might have some form of internal reference: i.e. that they might denote in a more or less direct way internal states, which are introspectively perceived by the first person and that the sensory experience of these states is part of the information our semantic representations consists in.

This view might have relevant consequences also with respect to our idea of concreteness, because it challenges the classical dichotomy between concrete words that are grounded in external information and abstract words that are understood primarily on the basis of linguistic information and it suggests that some abstract words are also grounded in some sort of sensory information, even though of an internal kind. In fact, by virtue of this 'grounding', these abstract words might be in some respects more similar to concrete words that to theoretical words. In particular, it is possible that the so-called concreteness effect (claimed to be responsible for the fact that concrete terms are processed more quickly than abstract terms ${ }^{7}$ and are learned earlier and more easily than abstract terms) does not only apply to words that rely on external sensory information. In fact, this might also take place in the case of words linked to internal sensory information, even though to a lesser extent since internal experience is less specific and less univocal than perceptual information. This is an empirical hypothesis which needs to be further investigated.

\section{Methodological Issues: Is There a Way to Assess Internal Grounding?}

The idea that introspective information is central to the representation of abstract concepts reshuffles the cards of the classical architecture of concrete and abstract words, challenging the standard picture of meaning according to which word representations are either grounded in external sensory information or in linguistic constructs, and suggests that words may also rely on internal sensory information.

Because of this radical perspective change, the measures psycholinguistics operationalized in order to distinguish between concrete and abstract words- the socalled 'concreteness' and 'imageability' constructs—could be obsolete since they were developed relying on the standard picture of meaning. However, the analysis we carried out in the previous part shows that this is not the case, at least not entirely. In fact, in $\S 3$ we suggested that the discrepancy (i.e. difference) between imageability and concreteness ratings offers an index of whether a word representation relies on internal information. However, this does not mean that these scales are perfectly adequate to differentiate between different kinds of non-concrete words and to establish which are mainly linguistic constructs and which are internally grounded. In this section we discuss some issues related to these two measures and their potential

${ }^{7}$ This means that the reaction times in tasks like lexical decision, word naming and recall are shorter for concrete than for abstract terms. For a recent review of the literature on this effect see e.g. Connell and Lynott (2012).

气 337249_1_En_3_Chapter-online $\square$ TYPESET $\square$ DISK $\square$ LE $\square$ CP Disp.:14/5/2015 Pages: 58 Layout: T1-Standard 
with respect to the new view. More specifically, we explain: (i) why the old constructs defined by Spreen and Schulz (1966) (concreteness) and Paivio et al. (1968) (imageability) that we used in our studies (this one as well as Dellantonio et al. 2014) are indeed appropriate for indicating whether words are internally grounded, even though they are not completely reliable and (ii) why on the contrary the new construct of concreteness defined by Brysbaert et al. (2014) is not at all reliable for our purposes.

First of all, as we have discussed extensively elsewhere, it is only a "methodological accident" that the imageability construct defined by Paivio is also a measure of the internal grounding of words. This accident is due to the fact that the instructions given to the participants on how to assign imageability ratings were misleading (Dellantonio et al. 2014):

Nouns differ in their capacity to arouse mental images of things or events. Some words arouse a sensory experience, such as a mental picture or sound, very quickly and easily, whereas others may do so only with difficulty (i.e., after a long delay) or not at all. The purpose of this experiment is to rate a list of words as to the ease or difficulty with which they arouse mental images. Any word which, in your estimation, arouses a mental image (i.e., a mental picture, or sound, or other sensory experience) very quickly and easily should be given a high imagery rating: any word that arouses a mental image with difficulty or not at all should be given a low imagery rating. Think of the words 'apple' or 'fact'. Apple would probably arouse an image relatively easily and would be rated as high imagery; fact would probably do so with difficulty and would be rated as low imagery. (Paivio et al. 1968, p. 4)

As we mentioned in the previous section, Paivio thought that imageability should closely resemble concreteness in all cases because in his view only concrete words had references that could be represented non-linguistically in the imagery system and were therefore easy to imagine (i.e. have high imageability ratings). For this reason, Paivio designed the instructions for the collection of imageability ratings primarily in terms of the ease or difficulty with which words arouse mental images of external things or events and the examples he uses are 'apple' and 'fact'.

However, in Paivio's view everything that can be perceived through the senses including e.g. smells, tastes, voices etc. is concrete. Thus, his notion of 'mental image' describes traces stored in memory of all types of external sensations (not only visual ones, but also auditory, olfactory, gustatory and haptic sensations should be taken into account). It is for this reason that in the instructions he specifies that all kind of sensory experience ("a sensory experience"; "other sensory experience") should be considered when making an imageability judgment. However, the request to estimate imageability depending on whether/how much a word arouses sensory experience without further specifications might have lead participants to assign their ratings on the basis of the ease/difficulty with which words aroused any kind of sensory experience stored in memory, including internal, body-related sensations. Thus, the fact that imageability appears to indirectly measure also the internal grounding of words (when compared with concreteness) is the side-effect of ambiguous instructions making the instructions instrumental in collecting biased ratings. 
Even though the instructions of imageability suggested evaluating the ease/ difficulty with which a word evoked any kind of sensory information including possibly internal experience, they are certainly biased towards an idea of imageability that is primarily visual and related to the ease/difficulty with which people can form a mental picture of the referent of a word. In fact, the term 'image' recalls quite strongly the idea of a visual picture. Thus, this instruction is certainly biased towards the sense of vision. This bias could represent a problem when we compare imageability with concreteness ratings. However, in the following part we will show that it does not. Specifically, we will argue that the instructions for concreteness also favor the sense of vision and that these two biases 'balance each other out'.

(i) The original instructions for concreteness ratings developed by Spreen and Schulz, (1966, p. 460) were as follows:

Nouns may refer to persons, places and things that can be seen, heard, felt, smelled or tasted or to more abstract concepts that cannot be experienced by our senses. The purpose of this experiment is to rate a list of words with respect to 'concreteness' in term of sense-experience. Any word that refers to objects, material or persons should receive a high concreteness rating; any word that refers to an abstract concept that cannot be experienced by the senses should receive a low concreteness rating. Think of the words 'chair' and 'independence'. 'Chair' can be experienced by our senses and therefore should be rated as high concrete; 'independence' cannot be experienced by the senses as such and therefore should be rated as low concrete (abstract).

According to the concreteness instructions something is concrete if it can be perceived through (at least one of) the senses. However, as it is has been already pointed out (Connell and Lynott 2012, p. 461), the examples mentioned in the second part of the definition ("objects, material or persons" as well as "chair" versus "independence") might have been misleading. In particular, they might have biased people to rely for their ratings (also) on a different idea of concreteness which resembles more closely the everyday understanding of the word 'concrete' and its dictionary definition, according to which 'concrete' means material or physical and an object is concrete only if it has a material composition. Since material objects are perceived mainly or primarily through vision and possibly through touch, people's ratings probably favored these senses over the others. However, since external material things are the ones people can more easily form a mental picture of, it can be predicted that for these material things there is an overlap between concreteness and imageability, independently of the sensory channel through which such material things are perceived.

We tested the hypotheses derived from the perusal of the instructions by performing two analyses. In the first analysis we checked whether there was a bias toward the sense of vision in Spreen and Schulz (1966) participants' ratings. We took into consideration the words included in the MRC database that have a concreteness rating $(\mathrm{N}=4260)$. We ordered them in descending order from those with the highest concreteness ratings to those with lower rating and determined if they were related to the sense of vision or to some other senses. All the words up to the 705th item, i.e. all words whose concreteness ratings exhibit a standard deviation higher than 1,17 , referred to material objects that could be perceived by vision. 
In the second analysis we checked whether the imageability ratings for words referring to senses other than vision did indeed correlate with the concreteness ratings for the same words. To do so we took into consideration the concreteness ratings of a list of words included in the MRC database denoting sounds, tastes and smells like 'sound', 'voice', 'rumor', 'salty', 'spicy', 'bitter', 'scent', 'odor', 'smell' etc. (words connected to touch like smooth, rough, soft, tender were not considered since they have more ambiguous meanings) and compared them with the mean concreteness ratings of other items in the corpus (purged of words occurring multiple times.) Things that can be perceived through senses other than sight $(\mathrm{N}=20)$ received mean concreteness and imageability ratings (432 and 460 respectively) comparable to those of the corpus as a whole ( $\mathrm{N}=4239 ; 439$ and 456 respectively). Moreover the correlation between imageability and concreteness for those words is significant $(\mathrm{r}=0.824, p<0.01)$. This comparison confirms that words denoting sounds, tastes and smells received only medium concreteness ratings and were therefore considered as less concrete than words denoting material objects that can be perceived through vision. However, the high correlation between concreteness and imageability ratings also points out that this class doesn't exhibit anomalies analogous to those observed with regard to emotion words, and it is therefore perfectly consistent with Paivio's view.

The results from our analyses can be summarized as follow. Despite what some studies maintain (e.g. Vigliocco et al. 2009; Connell and Lynott 2012), the ease/difficulty with which a word evokes a mental picture of a visual kind is not the only relevant aspect measured by imageability. Imageability also tracks the ease/difficulty with which a word evokes mental images aroused by senses other than vision. In fact, our analysis of words denoting sounds, tastes, and smells (we couldn't check touch) shows that - even though these imageability ratings tend to be lower than those for words denoting material objects-they are still highly correlated with concreteness ratings. Our data indicates that words denoting sounds, tastes, and smells are perceived as a bit less concrete and as a bit less imageable than words denoting material objects that can be seen. There is no inconsistency between the perceived concreteness and the perceived imageability in such cases (the correlation is significant); thus, the bias toward vision is not relevant for our conclusions concerning the viability of using the difference between imageability and concreteness as a measure of the internal grounding of a word.

Even though this bias is not relevant from our point of view, other authors have considered it extremely relevant and argue that many problems found in psycholinguistic studies in the past can be traced back to this bias. They therefore propose that a new concreteness measure should be defined to overcome this obstacle. One of the most influential studies with this aim is Connell and Lynott (2012). The authors' primary interest concerns the concreteness effect (mentioned at the end of §3), i.e. the behavioral advantages exhibited by words referring to concrete objects that are processed more quickly and accurately than abstract words. They point out that "despite their reputation as a textbook effect, concreteness effects do not always reliably emerge in semantic processing" (p. 453). In their view these problems are not due to the effect itself (which they consider indeed to be absolutely reliable), but 
rather to the means that are typically used to select the items for the experiments, i.e. the concreteness and the imageability measures. They think that both the concreteness and imageability ratings (which are often used interchangeably to assess the degree of concreteness versus abstractness of words) do not adequately capture the degree to which terms rely on perceptual experience because both the instructions for concreteness and imageability are affected by the biases we mentioned above. Specifically, while imageability is affected by a visual bias, concreteness is evaluated primarily on the basis of whether the word denotes a material object (thus, primarily on the basis of visual and haptic experience). Connell and Lynott do not consider the possibility that internal information might play a role with respect to the dichotomy abstract versus concrete and therefore suggest developing a new measure of concreteness which can substitute for both the old measure of concreteness and that of imageability. This new measure should rely on instructions in which participants are explicitly asked to consider each of the five perceptual modalities in turn: auditory, gustatory, haptic, olfactory and visual.

Also on the basis of Connell's and Lynott's arguments, a new set of concreteness ratings was recently collected using different instructions which drive the participant to assign concreteness ratings considering all the external senses (Brysbaert et al. 2014). Even though they are quite long, the instructions of this new database need to be reported in full since they determine the specific idea of concreteness participants relied on to assign the ratings:

Some words refer to things or actions in reality, which you can experience directly through one of the five senses. We call these words concrete words. Other words refer to meanings that cannot be experienced directly but which we know because the meanings can be defined by other words. These are abstract words. Still other words fall in-between the two extremes, because we can experience them to some extent and in addition we rely on language to understand them. We want you to indicate how concrete the meaning of each word is for you by using a 5-point rating scale going from abstract to concrete. A concrete word comes with a higher rating and refers to something that exists in reality; you can have immediate experience of it through your senses (smelling, tasting, touching, hearing, seeing) and the actions you do. The easiest way to explain a word is by pointing to it or by demonstrating it (e.g. to explain 'sweet' you could have someone eat sugar; to explain 'jump' you could simply jump up and down or show people a movie clip about someone jumping up and down; to explain 'couch', you could point to a couch or show a picture of a couch). An abstract word comes with a lower rating and refers to something you cannot experience directly through your senses or actions. Its meaning depends on language. The easiest way to explain it is by using other words (e.g. there is no simple way to demonstrate 'justice'; but we can explain the meaning of the word by using other words that capture parts of its meaning). Because we are collecting values for all the words in a dictionary (over 60 thousand in total), you will see that there are various types of words, even single letters. Always think of how concrete (experience based) the meaning of the word is to you. In all likelihood, you will encounter several words you do not know well enough to give a useful rating. This is informative to us too, as in our research we only want to use words known to people. We may also include one or two fake words which cannot be known by you. Please indicate when you don't know a word by using the letter $\mathrm{N}$ (or $\mathrm{n}$ ). So, we ask you to use a 5-point rating scale going from abstract to concrete and to use the letter $\mathrm{N}$ when you do not know the word well enough to give an answer. (Brysbaert et al. 2014, p. 906) 
These instructions rely explicitly on the standard picture of meaning according to which concrete terms are based on external perception while abstract terms rely on verbal information. Others than the instructions given by Spreen and Schulz (1966), these specify exactly how the contraposition between concrete and abstract should be understood, driving the participants to assign their ratings on the basis of the dichotomy perceivable through (at least one of) the senses versus linguistic construct.

The aim of the new instructions is to lead the participants to consider as concrete not only the terms denoting material objects (perceivable through the sight and through the touch), but also words whose denoting properties that are perceivable only through other senses (e.g. words denoting sounds, odors, tastes etc.). However, the analysis carried out by the authors on the ratings of the new database confirms the bias toward material objects: also with the new instructions people tended to assign a higher degree of concreteness to material objects:

The high correlation between our ratings and those included in the MRC database ( $\mathrm{r}=$ .92) attests to both the reliability and the validity of our ratings [...]. At the same time, the high correlation shows that the extra instructions we gave for the inclusion of nonvisual and action related experiences, did not seem to have much impact. Gustatory strength was not taken into account and auditory strength even correlated negatively, because words such as "deafening" and "noisy" got low concreteness ratings (1.41 and 1.69 respectively) but high auditory strength ratings (5.00 and 4.95). Apparently, raters cannot take into account several senses at the same time. (Brysbaert et al. 2014, p. 908)

Brysbaert and collaborators report that they found a "high correlation" between their ratings and those included in the MRC database. However, this overall correlation hides some differences that in our view are relevant. Indeed, if one considers specifically the words that are most problematic with respect to Paivio's theory and more generally with respect to the standard picture of meaning-i.e. those denoting $\mathrm{x}$-ceptive states (i.e. affective, interoceptive and proprioceptive states)—and compares the concreteness ratings of these words in the MRC database and in Brysbaert and collaborators' collection, the result is quite different. Indeed, we confronted the concreteness ratings of the x-ceptive words selected for our previous study (Dellantonio et al. 2014) in the two databases and found that whereas their mean concreteness was 428 in the MRC database, it was 492 in the Brysbaert and colleagues' database, and that the 64 points of difference are statistically significant, $\mathrm{t}(48)=6.1$, $p<0.001$. The fact that concreteness ratings for these words increased is extremely relevant since it results in the reduction of the difference between imageability and concreteness values which in our view indicate the link between a word and information of internal kind. The reason why the concreteness ratings of at least some relevant items like the $\mathrm{x}$-ceptive words are higher in the new collection with respect to MRC lies in the instructions given to the participants and especially in two aspects of them.

First of all, these instructions lead participants to 'externalize' the criteria used to represent word meanings. The example of 'sweet' is highly significant in this respect: "The easiest way to explain a word is by pointing to it or by demonstrating it (e.g. to explain 'sweet' you could have someone eat sugar.)". 'Sweet' denotes a 
flavor. If we consider as concrete all words denoting something perceivable with one of the external senses, then the word 'sweet' is certainly concrete. However, its concreteness is not due to the fact that we can imagine the visual picture of somebody eating sugar, as suggested by the example, but it is due to the fact that we can directly perceive this sweetness through our sense of taste. If we observe somebody else eating something we never tasted before using our own papillae, we cannot know if it is sweet or not. In this sense, Brysbaert and collaborators' instructions are misleading since-in order to pursue the aim of making flavors appear more concrete to the participants and more close to the experience of material objects-they present the sensation of taste in terms of the visual perception of someone else eating. In this way, these instructions disregard the difference between internal (subjective) and external experience-i.e. between tasting 'sweet' directly and seeing somebody else eating something sweet-leading people to reinterpret their internal experience entirely in terms of external experience. ${ }^{8}$ This is the reason why-following this instructions - people might have assigned a higher concreteness rating to all kind of internal sensations, interpreting interoceptive, exteroceptive or affective states more or less like tastes, sounds or smells. In the spirit of these instructions, since we can easily imagine the visual picture of someone moving, or making a suffering face because of the pain, or expressing his/her happiness by making a happy face, 'movement', 'pain' and 'happiness' must be considered as (fairly) concrete words. However, in this way the difference between concreteness and imageability disappears together with the difference between internal and external sensory experience.

Secondly, the instructions implicitly link concrete words with ostension, suggesting that a world is concrete if we can point to what it denotes. At the same time they suggest that the concreteness of actions should also be assessed this way: "to explain 'jump' you could simply jump up and down or show people a movie clip about someone jumping up and down". As in the case of 'sweet', also 'jump' leads to an externalized interpretation of the experience we use to understand words: we are not asked to think of the sensations we subjectively experience when we jump, but we are asked to take an external perspective and to picture someone jumping. In this way the participants are led to disregard the dynamical, first person information they have on the active performance of the action and to think of an action as they observe it externally when it is performed by someone else. Thus, instructions lead the participants to think of actions as if they were objects that can be perceived only through external observation. This externalization of actions produces the same effect discussed in

${ }^{8}$ The sense of taste belongs to the traditional five external senses, however, traditionally it is considered the most internal among the external senses in opposition to vision, which is considered as the external sense par excellence. While vision easily allows us to identify intersubjectively the source of the stimulation and is therefore considered to be also the most objective and real among the external senses, the sense of taste is the less objective because it is impossible to verify intersubjectively what a person is experiencing. For this reason, the notion of 'taste' has been generalized to indicate subjective judgments that cannot be disputed and are therefore potentially arbitrary (e.g. "it is a matter of taste"). For some philosophical reflections on the sense of taste in this vein see e.g. Arendt (1978, 1982). 
the previous step: all words describing actions are evaluated as being concrete. Since their concreteness ratings are higher, the difference from their imageability becomes minimal and it becomes impossible to assess whether proprioceptive information plays a role with respect to the understanding of these words.

This externalization of the sensations and of actions makes this new database for concreteness (i) methodologically incommensurable with respect to MRC (and this in spite of the high correlation between the ratings reported in Brysbaert et al. (2014) and those included in the MRC database) and (ii) inadequate to assess a threedimensional view of the difference between abstract and concrete in terms of which word meanings reflect different degrees of involvement of different kinds of external and internal sensory information as well as linguistic information.

As we showed above, the old concreteness and imageability scales can provide some useful indications to assess the different degrees of involvement of external, internal and linguistic information. However, they are still much too inaccurate and a more specific measure would be needed to assess abstractness and concreteness according to this new three-dimensional perspective. Ideally, such a measure should — among other things—include a distinction between grammatical categories (in particular between nouns and verbs) since on the basis of our discussion of the externalization of actions, one could hypothesize that verbs rely more heavily on internal (i.e. proprioceptive) information than the corresponding nouns, and specifically that e.g. 'to run' might evoke motor information on the performance of the action, while 'run' as a noun might evoke a more passive and externalized representation of a run.

\section{Concluding Remarks}

This study challenges the standard picture of meaning according to which words either rely on external sensory experience or are linguistic constructs and argues for the view that word meanings reflect different degrees of involvement of different kinds of external and internal sensory information as well as linguistic information. The paper addresses not only the theoretical aspects of this issue, but also its methodological consequences. In this regard, we specifically discuss whether the measures of concreteness and imageability used in the standard theory which opposed concrete and abstract words, might or might not continue to be useful since they allow us to assess the contribution of external and internal information. In the context of this discussion we suggest that, even though the classical constructs of concreteness and imageability might in fact be used as a joint measure to evaluate, at least approximately, whether a word representation relies on internal information, a more specific measure is needed for this aim.

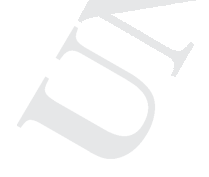

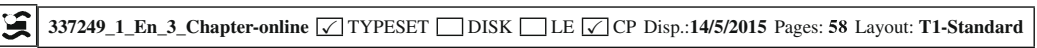




\section{References}

Achinstein, P. (1965). The problem of theoretical terms. American Philosophical Quarterly, 2(3), 193-203.

Ackrill, J. (1963). Aristotle. Categories and de interpretatione. Oxford: Clarendon Press.

Altarriba, J., \& Bauer, L. (2004). The distinctiveness of emotion concepts: A comparison between emotion, abstract, and concrete words. The American Journal of Psychology, 117(3), 389-410.

Altarriba, J., Bauer, L., \& Benvenuto, C. (1999). Concreteness, context availability, and imageability ratings and word associations for abstract, concrete, and emotion words. Behavior Research Methods, 31(4), 578-602.

Arendt, H. (1978). The life of mind. New York: Harcourt.

Arendt, H. (1982). Lectures on Kant's political philosophy. Chicago: Chicago University Press.

Barsalou, L. W. (1999). Perceptual symbol systems. Behavioral and Brain Sciences, 22(4), 577-660.

Berthoz, A. (2000). The brain's sense of movement. Cambridge: Harvard University Press.

Brown, D. J. (2006). Descartes and the passionate mind. Cambridge: Cambridge University Press.

Barsalou, L. W. (2008). Grounded cognition. Annual Review of Psychology, 59, 617-645.

Barsalou, L. W., \& Wiemer-Hastings, K. (2005). Situating abstract concepts. In D. Pecher \& R.

Zwaan (Eds.), Grounding cognition: The role of perception and action in memory, language, and thought (pp. 129-163). New York: Cambridge University Press.

Brysbaert, M., Warriner, A. B., \& Kuperman, V. (2014). Concreteness ratings for 40 thousand generally known English word lemmas. Behavior Research Methods. 46(3), 904-911. doi:10. 3758/s13428-013-1403-5.

Barsalou, L. W., Niedenthal, P. M., Barbey, A. K., \& Ruppert, J. A. (2003). Social embodiment. In B. H. Ross (Ed.), The psychology of learning and motivation. Advances in research and theory. Amsterdam: Academic Press.

Carnap, R. (1956). The methodological character of theoretical concepts. In H. Feigl \& M. Scriven (Eds.), Minnesota studies in the philosophy of science I (pp. 38-76). Minneapolis: University of Minnesota Press.

Cook, J. W. (1965). Wittgenstein on privacy. The Philosophical Review, 74(3), 281-314.

Coltheart, M. (1981). The MRC psycholinguistic database. The Quarterly Journal of Experimental Psychology Section A: Human Experimental Psychology, 33(4), 497-505.

Cruse, A. (2000). Meaning in language. An introduction to semantics and pragmatics. Oxford: Oxford University Press

Craig, A. D. (2003). Interoception: The sense of the physiological condition of the body. Current Opinion in Neurobiology, 13(4), 500-505.

Craig, A. D. (2009). How do you feel-now? the anterior insula and human awareness. Nature Reviews Neuroscience, 10(1), 59-70.

Craig, A. D. (2010). The sentient self. Brain, Structure \& Function, 214(5-6), 563-577.

Candlish, S., \& Wrisley, G. (2012) "Private language", The stanford encyclopedia of philosophy (Summer 2012 Edition), Edward N. Zalta (Ed.), http://plato.stanford.edu/archives/sum2012/ entries/private-language/.

Connell, L., \& Lynott, D. (2012). Strength of perceptual experience predicts word processing performance better than concreteness or imageability. Cognition, 125(3), 452-465.

Damasio, A. (1999). The feeling of what happens. Body and emotions in the making of consciousness. Orlando: Harcourt.

Dixon, T. (2003). From passion to emotions. The creation of a secular psychological category. Cambridge: Cambridge University Press.

Damasio, A. (2010). Self comes to mind. Constructing the conscious brain. New York: Pantheon. Dellantonio, S., \& Pastore, L. (2006). What do concepts consist of? The role of geometric and proprioceptive information in categorization. In P. Hanna, A. McEvoy, \& P. Voutsina (Eds.), An anthology of philosophical studies (pp. 91-102). Athens: Athens Institute for Education and Research. 
Dellantonio, S., Mulatti, C., Pastore, L., \& Job, R. (2014). Measuring inconsistencies can lead you forward: Imageability and the x-ception theory. Front Psychology. doi:10.3389/fpsyg.2014. 00708.

Ekman, P. (1994). Moods, emotions and traits. In E. Ekman \& R. Davidson (Eds.), The nature of emotion: Fundamental questions (pp. 56-58). Oxford: Oxford University Press.

Ekman, P. (1999). Basic emotions. In T. Dalgleish, \& M. Power (Eds.), Handbook of cognition and emotion (pp. 45-60). Sussex, UK: Wiley.

Ekman, P., Sorenson, E., \& Friesen, W. (1969). Pan-cultural elements in facial displays of emotion. Science, 164(3875), 86-88.

Goldman, A. (1993). Philosophical application of cognitive science. Boulder: Westview Press.

Gibbs, R. W., Jr. (1994). The poetics of mind: Figurative thought, language, and understanding. New York: Cambridge University Press.

Johnson, M. (1987) The body in the mind: The bodily basis of meaning, imagination, and reason. Chicago: University of Chicago Press.

Kripke, S. (1982). Wittgenstein on rules and private language. Oxford: Blackwell.

Knorrp, W. M. (2003). How to talk to yourself or Kripke's Wittgenstein solitary language argument and why it fails. Pacific Philosophical Quarterly, 84(3), 215-248.

Kassam, K., Markey, A., Cherkassy, V., Loewenstein, G., \& Just, M. (2013). Identifying emotions on the basis of neural activation. PLoS ONE, 8(6), e66032.

Kousta, S., Vigliocco, G., Vinson, D., Andrew, A., \& Del Campo, E. (2011). The representation of abstract words: Why emotion matters. Journal of Experimental Psychology: General, 140(1), 14-34.

Lakoff, G. (1987) Women, fire, and dangerous things: What categories reveal about the mind. Chicago: University of Chicago Press.

Lebuffe, M. (2010). The anatomy of the passions. In O. Koistinen (Ed.), The cambridge companion to Spinoza's ethics (pp. 188-222). Cambridge: Cambridge University Press.

Lakoff, G. \& Johnson, M. (1980) Metaphors we live by. Chicago: University of Chicago Press.

Lakoff, G. \& Turner, M. (1989) More than cool reason: A field guide to poetic metaphor. Chicago: University of Chicago Press.

Nigel, T. (2013). Mental imagery. In E. Zalta (Ed.), The stanford encyclopedia of philosophy. http:// plato.stanford.edu/archives/fall2013/entries/mentalimagery/.

Paivio, A. (1965). Abstractness, imagery, and meaningfulness in paired-associate learning. Journal of Verbal Learning and Verbal Behaviour, 4(1), 32-38.

Plutchik, R. (1980). A general psychoevolutionary theory of emotion. In R. Plutchik \& H. Kellerman (Eds.), Emotion: Theory, research, and experience: Vol. 1. Theories of emotion (pp. 3-33). Academic Press: New York.

Paivio, A. (1986). Mental representations. A dual coding approach. Oxford: Oxford University Press.

Papineau, D. (1996). Theory-dependent terms. Philosophy of Science, 63(1), 1-20.

Prinz, J. (2002). Furnishing the mind: Concepts and their perceptual basis. Cambridge: MIT Press.

Prinz, J. (2004). Gut reactions. A perceptual theory of emotion. Oxford: Oxford University Press.

Paivio, A. (2007). Mind and its evolution: A dual coding theoretical approach. Mahwah: Erlbaum.

Paivio, A., Yullie, J., \& Madigan, S. (1968). Concreteness, imagery, and meaningfulness values for 925 nouns. Journal of Experimental Psychology. Monograph Supplement, 76(1), 1-25.

Reizenzein, R. (2009). Emotional experience in the computational belief-desire theory of emotion. Emotion Review, 1(3), 214-222.

Schroeder, S. (1998). Das Privatsprachen-Argument. Wittgenstein über Empfindung und Ausdruck. Paderborn: F. SchöningVerlag.

Spreen, O., \& Schulz, R. (1966). Parameters of abstractions, meaningfulness and pronunciability. Journal of verbal learning and Verbal Behaviour, 5(5), 459-468.

Tomkins, S. (1962). Affect, imagery, consciousness: Vol. I: The positive affects. New York: Springer. Tomkins, S. (1963). Affect, imagery, consciousness: Vol. II: The negative affects. New York: Springer. 
940 Vigliocco, G., Meteyard, L., Andrews, M., Kousta, S. (2009). Toward a theory of semantic representation. Language and Cognition, 1(2), 219-247.

942 Wittgenstein, L. (1953). Philosophical investigations, translated by G. E. M. Anscombe, Oxford: Blackwell (3rd ed.) 1967.

944 Wilson, M. (1988). The MRC psycholinguistic database: Machine readable dictionary, version 2. 945 Behavioural Research Methods, Instruments and Computers, 20(1), 6-11. 